Original paper

\title{
Beam quality and dose perturbation of 6 MV flattening-filter-free linac
}

\author{
P. Tsiamas ${ }^{\text {a,b }}$, E. Sajo ${ }^{\text {c }}$, F. Cifter ${ }^{\text {c }}$, K. Theodorou ${ }^{\text {b }}$, C. Kappas ${ }^{\text {b }}$, M. Makrigiorgos ${ }^{\text {a }}$, \\ K. Marcus ${ }^{\mathrm{a}, *}$, P. Zygmanski ${ }^{\mathrm{a}, *}$ \\ ${ }^{a}$ Brigham and Women Hospital and Harvard Medical School, Boston, MA 02115, USA \\ ${ }^{\mathrm{b}}$ Medical Physics Department, Medical School, University of Thessaly, Larisa 4100, Greece \\ ${ }^{\mathrm{c}}$ University of Lowell Massachusetts, Department of Physics and Applied Physics, Medical Physics Program, Lowell, MA 01854, USA
}

\section{A R T I C L E I N F O}

\section{Article history:}

Received 16 August 2012

Received in revised form

7 January 2013

Accepted 19 February 2013

Available online 18 March 2013

\section{Keywords:}

Monte Carlo

Flattening-filter-free

Dose enhanced radiation therapy

Photon fluence

\begin{abstract}
A B S T R A C T
The aim of this study is twofold: (a) determination of the spectral differences for flattening-filter-free (FFF) versus standard (STD) linac under various clinical conditions, (b) based on an extensive list of clinically important beam configurations, identification of clinical scenarios that lead to higher macroscopic dose perturbations due to the presence of high- $Z$ material. The focus is on dose enhancement due to contrast agents including high- $Z$ elements such as gold or gadolinium.

EGSnrc was used to simulate clinical beams under various irradiation conditions: open/IMRT/spitIMRT fields, in/out-off-field areas, different depths and field sizes. Spectra were calculated and analyzed for about 80 beams and for a total of 480 regions. Quantitative differential effects in beam quality were characterized using energy-dependent and cumulative dose perturbation metrics.

Analysis of the spectral database showed that even though the general trends for both linacs (FFF/STD) were the same, there were crucial differences. In general, the relative changes between different conditions were smaller for FFF spectra. This was because of the higher component of low-energy photons of the FFF linac, which already lead to higher dose enhancement than for the STD linac (photon energies were more "uniformly" distributed for FFF spectra and henceforth their perturbation resulted in lesser relative changes). For out-of-field FFF spectra and split-IMRT fields the strongest enhancement were observed ( $\sim 25$ and $\sim 5$ respectively). Different spectral scenarios lead to different dose enhancements, however, they scale with the higher effective- $Z$ of the materials and were directly related to the lower range of the spectra $(<200 \mathrm{keV})$.
\end{abstract}

(c) 2013 Associazione Italiana di Fisica Medica. Published by Elsevier Ltd. All rights reserved.

\section{Introduction}

Characterization of therapeutic and imaging linac beam spectra is of common interest through the scientific community as it is important for a wide range of applications [1-7]. The linac beam energy at a point depends on field size, lateral location with respect the central axis, depth, presence of heterogeneities, and the treatment technique used. Spectral variations play a role in dosimetry when energy-dependent detectors, such as TLDs, films, diode/ion chamber arrays are used [8]. Such changes affect the response of these dosimeters, which have to be calibrated in conditions as close as possible to the conditions they are being used, and in the case of mixed spectral content beams they may result in an inaccurate dose measurement. In addition, structural and design differences in the

\footnotetext{
* Corresponding authors. Tel.: +1 6178694504.

E-mail addresses: ptsiamas@lroc.harvard.edu (P. Tsiamas), kmarcus@ lroc.harvard.edu (K. Marcus), pzygmanski@lroc.harvard.edu (P. Zygmanski).
}

medical equipment or delivery techniques can lead to substantially different dosimetric beam properties.

One of the recent examples of these design and structural differences was the use of linacs operating in flattening-filter-free mode. Using Monte Carlo (MC) simulation techniques a number of investigators have studied the beam properties and characteristics of flattening-filter-free linacs [9-21]. Removal of the flattening filter increases the lower energy component of the spectrum as this is the major beam hardening source of the linac head. That change can significantly alter the beam spectrum in different regions of the field and outside of it and has not been investigated from that perspective besides a few examples presented in recent studies [12,17]. Moreover, removal of the flattening filter may also change other clinically important properties of the beam. For instance, it can lead to a dramatic difference of electron spectra of a Varian linac at the surface of a water phantom [9] and move maximum dose $\left(d_{\max }\right)$ shallower by $10-20 \%$ depending on the field size [11]. In the last paper it was calculated that the effective energy of an unflattened beam drops by about $2 \mathrm{MV}$ (from $6 \mathrm{MV}$ to 
$4 \mathrm{MV}$ for a Varian linac) compared to a beam shaped by flattening filter. This means that a PDD of FFF linac having the same nominal energy (6 MV) corresponds to a PDD of a linac with flattering filter and nominal energy of about $4 \mathrm{MV}$.

So far there has been only a few studies published showing spectral changes of clinical photon beams, and in particular there is a general lack of comprehensive information about FFF linacs, which might be suitable for contrast enhanced therapies. The main focus of the past studies had been the exploration of the problem of dose variations when energy-depended dosimeters were used. These studies compared photon spectra for different field sizes, depths and treatment techniques [1-3]. Apart from a recent study by Scarboro et al. [2], which presented an extensive investigation for different cases only for STD linac, the rest of the studies showed very limited examples of spectra.

One of the new areas of radiation therapy and imaging in which spectral differences are of great importance is dose enhanced radiation therapy with high- $Z$ contrast agents and nanoparticles (such as iodine or gadolinium molecules, or gold nanoparticles). The past few years have seen an acceleration of research exploring these techniques, which exploit the increased photoelectric absorption when materials of high- $Z$ content are taken up by various tissues or cells [22-27]. Some of the studies concluded that dose enhancement is too low and can be practically neglected [27]. Others calculated a dose enhancement increase by a factor of $\sim 8$ [24]. Investigations at this area are facing both experimental and theoretical challenges (e.g. Monte Carlo simulations are highly sensitive to specific normalization schemes and the phantom setup at the sub-mm scale). Changes of the lower energy portion of the spectrum (e.g. $<300 \mathrm{keV}$ ) due to different irradiation conditions, in the regions where high- $Z$ material is present, might have a large effect on the dose deposition at the microscopic scale while preserving the macroscopic dose level at its prescription value. Knowledge of the effective beam quality (effect of a high- $Z$ material on the spectral quality of a clinical beam) for these recently explored therapies is essential both for imaging and treatment delivery using low concentrations of high- $Z$ elements.

Despite the recent interest in flattening-filter-free linac beams, there is a lack of systematic comparison of how the energy spectra will change among different clinical scenarios. The aim of this paper is to present a comprehensive spectral database and determine the qualitative and quantitative differences of clinical spectra when the linac operates in flattening-filter-free mode (FFF) for an extensive list of clinically important beams. A secondary aim of the current paper is to identify possible clinical scenarios that might change the lower part of the spectrum (e.g. below $300 \mathrm{keV}$ ), area which is associated with dose enhancement in radiation therapy and contrast enhancement in imaging.

\section{Materials and methods}

\section{Monte Carlo simulation}

Photon spectra were calculated for a Varian 6 MV linac using the EGSnrc Monte Carlo (MC) computer model [28]. The accelerator head was simulated with and without flattening filter, for both in and out-of-field areas. For FFF linac simulations flattening filter was removed from the simulation and a $2 \mathrm{~mm}$ copper plate was placed in the position of the flattening filter. This was based on previous publications [9] but also on observation by the authors during the process of the flattening filter removal. Briefly, the following simulation parameters were used. Cut-off energy for electrons (ECUT) and photons (PCUT) was set to $0.711 \mathrm{MeV}$ and $0.01 \mathrm{MeV}$ respectively. Directional Bremstrahlung Splitting (DBS) was selected for Bremstrahlung photons (Brems splitting number $=2000$ ) with splitting and field radius set at $40 \mathrm{~cm}$, in order to cover a large area in and out-of-field. All EGSnrc default parameters and options were used, except for options "Bound Compton scattering", "Photoelectron angular sampling" and "Atomic relaxations" which were set to on mode. For "Brems angular sampling" and "Pair angular sampling" options Koch-Motz (KM) distribution was chosen. Electron beam standard deviation $\left(\sigma_{x}, \sigma_{y}\right)$ values were set to $0.08 \mathrm{~cm}$ ( $X$ direction) and $0.16 \mathrm{~cm}$ ( $Y$ direction) while electron beam energy was set to $6.5 \mathrm{MeV}$ and angular spread of electron beam $\sigma_{\theta}$ was $0^{\circ}$ respectively. Details about the MC model and the simulation parameters are included in a previous study [21]. The MC model included the multileaf collimator (MLC - 120 leaves) by using the DYNVMLC module, which permits the simulation of dynamic fields. All simulations were performed using 20 million initial histories. That provided a more direct comparison between different spectra and different dose values as both of them are normalized to the number of initial histories (incident particles). The number of particles in the produced phase spaces ranged from around 10 million to 1.1 billion depending on the simulation conditions. A total of 80 beams and about 480 spectral cases ( 4 field sizes $3 \times 3-14 \times 28 \mathrm{~cm}^{2}, 4$ depths $0-20 \mathrm{~cm}, 2$ linac types FFF/STD, open beam (OB) versus IMRT and split-IMRT, 4 MLC gap sizes $1-20 \mathrm{~mm}$, 3 different areas of interest per case in/out-of-field). For the extraction of the spectra BEAMDP tool of the EGSnrc code was used.

For dose calculation the DOSXYZnrc code was used. Input in the DOSXYZnrc was the produced at the surface of the phantom phase space of each case (e.g. IMRT, field size, etc.). The same sets of EGSnrc parameters were used for the BEAMnrc simulation while 500 million-1 billion histories were simulated depending on the case. The simulated phantom was $100 \times 100 \times 40 \mathrm{~cm}^{3}$ size, placed at a source-to-surface distance (SSD) of $100 \mathrm{~cm}$. A uniform voxel size of $1.0 \times 1.0 \times 0.5 \mathrm{~cm}^{3}$ was used in the entire phantom.

\section{Clinical scenarios for photon spectra}

Energy fluence photon spectra were obtained for different cases in order to investigate the potential differences of FFF linac versus STD. For that reason the following parameters were studied: field size, depth, distance from the central axis of the field (CAX), IMRT versus open beam fields (OB), and split-IMRT fields.

Spectra were calculated in air at the surface of the phantom and inside a homogenous water phantom. For the water phantom the scalar fluence was calculated at depths of $2 \mathrm{~cm}, 10 \mathrm{~cm}$ and $20 \mathrm{~cm}$. Simulated field sizes were $3 \times 3 \mathrm{~cm}^{2}, 5 \times 5 \mathrm{~cm}^{2}$, $10 \times 10 \mathrm{~cm}^{2}$ and $14 \times 28 \mathrm{~cm}^{2}$. Dynamic sweeping gaps (SG) representing IMRT fields of various MLC transmission content were simulated only for $10 \times 10 \mathrm{~cm}^{2}$ and $14 \times 28 \mathrm{~cm}^{2}$ field sizes. For the $10 \times 10 \mathrm{~cm}^{2}$ field the gap sizes used were $10 \mathrm{~mm}$ and $20 \mathrm{~mm}$, and for $14 \times 28 \mathrm{~cm}^{2}$ field the selected gaps were $1 \mathrm{~mm}$, $5 \mathrm{~mm}, 10 \mathrm{~mm}$ and $20 \mathrm{~mm}$.

Scalar fluence as a function of energy $[\Phi(E)]$ and scalar energy fluence as a function energy $[\Psi(E)]$, were calculated for different areas inside and outside of the beam over a $1 \times 1 \mathrm{~cm}^{2}$ region of interest. For each phase space depth, the spectra were calculated at three different locations of ROI: on the CAX, $2 \mathrm{~cm}$ out-of-field and $4 \mathrm{~cm}$ out-of-field (Fig. 1a). The spectra were analyzed in various forms: raw data without normalization, data normalized to the total area under the spectral curve, and raw spectra divided by the average dose within the particular region of interest.

In order to reduce the MC variance in out-of-field zones the following technique was used: In the case of square fields spectra were calculated at four centrally symmetric areas having the same distance $(c)$ from the central axis and located at coordinates $(0, c)$, 

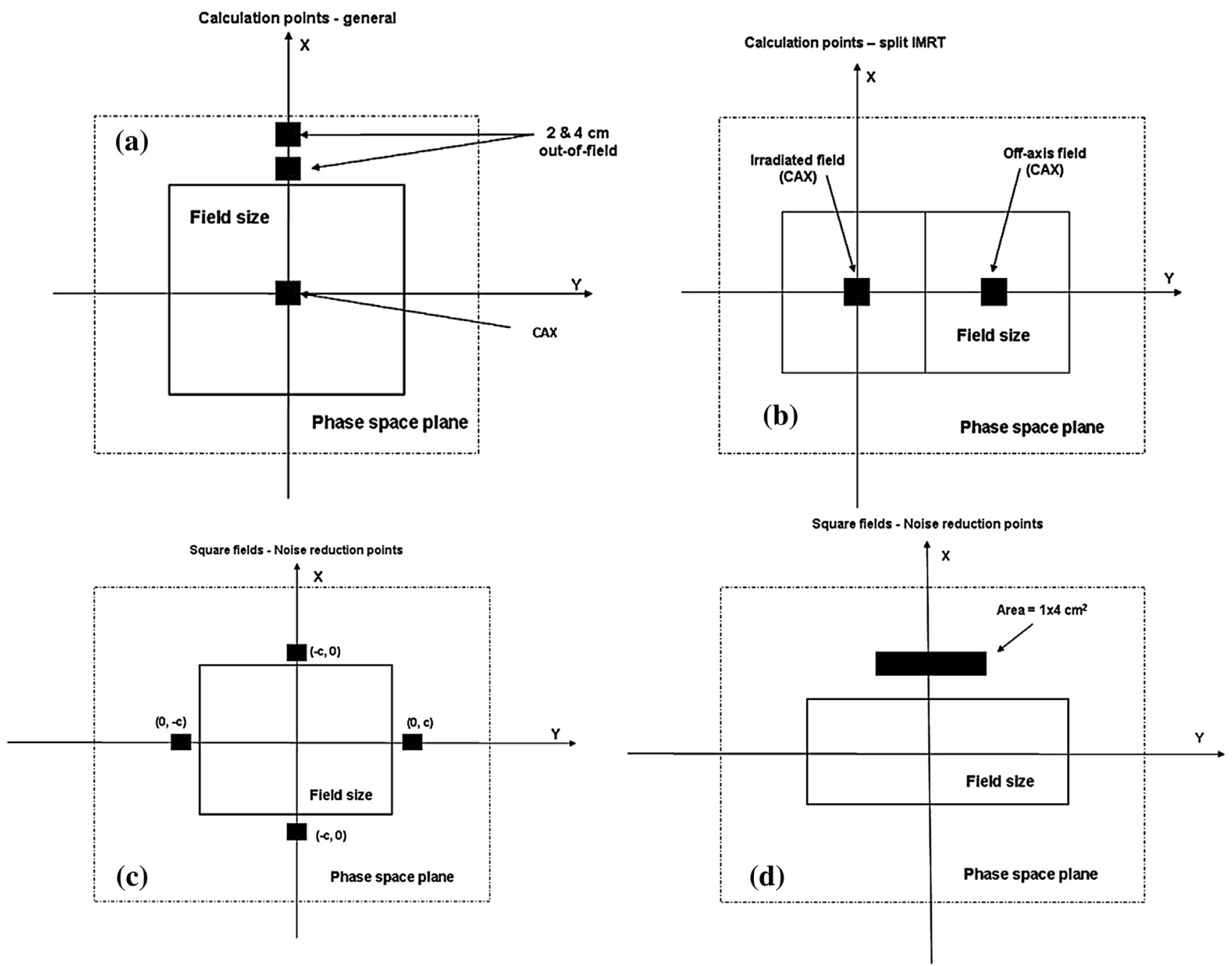

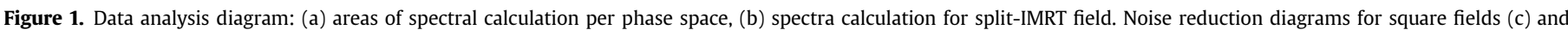
rectangular fields $(\mathrm{d})$.

$(0,-c)$ and $(c, 0),(-c, 0)$. The spectrum used for the analysis was the average one of the spectra calculated at these four areas (Fig. 1c). For the $14 \times 28 \mathrm{~cm}^{2}$ field the calculation area was increased from $1 \times 1 \mathrm{~cm}^{2}$ to $1 \times 4 \mathrm{~cm}^{2}$ (Fig. $1 \mathrm{~d}$ ).

In clinical practice when the treated tumor volumes are large (e.g. pelvis, or head and neck) IMRT fields might split into two or even three separate subfields to be able to deliver IMRT (Fig. 1b). One of our goals is to calculate the difference between the spectrum of a single large unsplit IMRT field and of spectral change due to splitting of the IMRT field. Splitting IMRT fields results in relatively large contribution of out-of-field dose to the target regions which is typically undesirable in standard treatments, but which might have beneficial effect in dose enhancement therapy.

In Fig. 1b MLC leaves are moving along $x$-axis, the directly irradiated subfield is the one on the left while the out-of-field one is the right one. Therefore, in such cases the tumor located at the center of the left subfield after irradiation of both subfields (left and right) will have received direct dose from the irradiation of the left field and indirect dose from the scatter radiation coming from the second subfield. So a comparison of two spectra was done: the one obtained for a single IMRT field $\left(10 \times 10 \mathrm{~cm}^{2}\right.$ field, $\left.S G=10 \mathrm{~mm}\right)$ and one resulting from the irradiation of two similar abutting $10 \times 10 \mathrm{~cm}^{2}$ IMRT fields $(\mathrm{SG}=10 \mathrm{~mm})$.
The incident photon energy ranged from $10 \mathrm{keV}$ to the maximum nominal energy of the beam (6.5 MeV). Energy binning was linear with $65 \mathrm{keV}$ increments for the whole spectrum. Spectra planes were placed at the center of the dose voxel and each spectrum was also correlated to its dose value in analysis of the data.

\section{Spectrum-weighted dose metrics}

The spectrum may change significantly depending on the irradiation and beam conditions. Therefore, the impact of the beam spectrum on the dose depends on the specific application. In view of the goals set in this work, in Appendix A we define two metrics that will be helpful in the assessment of such impact: an energydependent dose perturbation (dose enhancement or suppression) factor, $m(E)$, and an effective dose perturbation factor, $M$ for the entire spectrum. Similar metrics has been used in the past [29]. Using these two metrics and the differences between them for various irradiation conditions (e.g. $M$ (cond) $-M\left(\right.$ cond $\left._{\mathrm{ref}}\right), m(E$, cond $)-m\left(E\right.$, cond $\left.\left.\mathrm{ref}_{\mathrm{r}}\right)\right)$, we discuss the impact of spectral changes on the dosimetry of selected materials such as scintillators (gadolinium oxysulfide $-\mathrm{Gd}_{2} \mathrm{O}_{2} \mathrm{~S}$ ) and gold $(\mathrm{Au}$ ) employing mass-energy absorption coefficients available from NIST tables [30]. 


\section{Results}

\section{Spectral comparison}

In this section spectral comparison will be presented for the various irradiation conditions selected. The spectra were divided into three main categories: inside the field, outside of field and IMRT. All the plots of this section are showing normalized particle fluence (normalization to the total area) versus energy.

\section{Spectral variations inside the irradiated field}

Figure 2a shows spectral changes with depth for FFF versus STD linac. For both linac types there was a shifting of the spectrum towards lower energies with increasing depth. Comparison between FFF and STD linac showed that FFF was softer compared to STD spectrum while for FFF spectrum the intermediate area, between $200 \mathrm{keV}$ and $1 \mathrm{MeV}$, was almost flat as can be observed from the plots. Although the lower energy area (e.g. energies smaller than $200 \mathrm{keV}$ ) of an FFF linac is larger (by comparison of the area below each curve) than for an STD linac the relative increase of this area with depth (e.g. from depth of $2 \mathrm{~cm}$ to depth of $20 \mathrm{~cm}$ ) is less for the unflattened beam compare with the one with flattening filter. That means that the lower part of the spectrum undergoes a larger difference with depth in the case of an STD linac.

Comparison of the spectra for the same depth $($ e.g. $10 \mathrm{~cm})$ but for different field sizes at CAX showed shifting of the spectrum towards lower energies as field size increased (Fig. 2b). The magnitude of the total area below the curves for energies below $200 \mathrm{keV}$ increased by up to a factor of 2 as field size was increased from
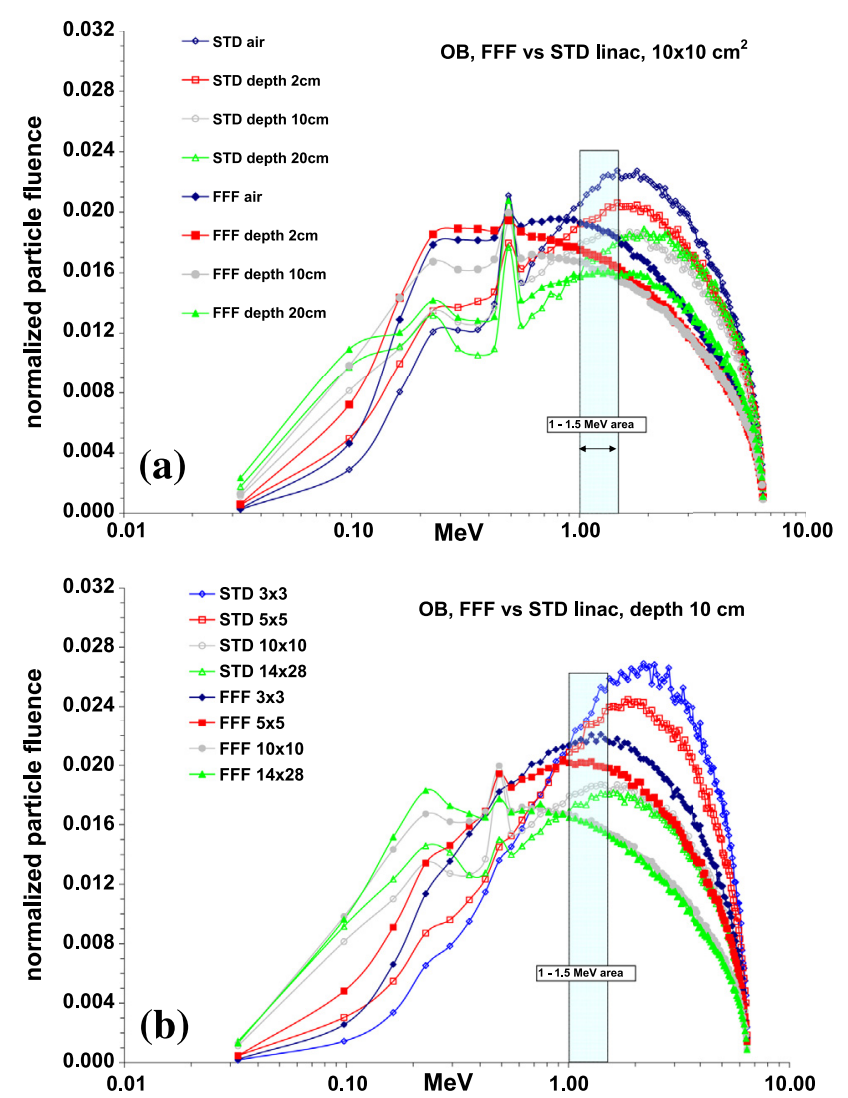

Figure 2. Spectral variation comparison of FFF versus STD linac inside the field for different depths $\left(\mathrm{a}-\right.$ field size $=10 \times 10 \mathrm{~cm}^{2}$ ) and different field sizes (b depth $=10 \mathrm{~cm}$ ) for open beam $(\mathrm{OB})$. Spectra shown are normalized to the total area.
$3 \times 3 \mathrm{~cm}^{2}$ to $10 \times 10 \mathrm{~cm}^{2}$ (Fig. 2b). The energy and the height where the peak of the $\Psi(E)$ spectra was noticed varied with case. This is expected as different beams have different spectral quality. Depending on the conditions, in the case of STD the spectral maximum shifted from approximately $1.5 \mathrm{MeV}$ to $2 \mathrm{MeV}$. Trends were similar for the FFF spectra but here the maximum shifted from approximately $1 \mathrm{MeV}$ to $1.5 \mathrm{MeV}$. The FFF spectrum was softer, with a shift in the energy of its peak by $(0.5 \mathrm{MeV})$. Another difference between FFF and STD spectrum was that FFF spectrum was practically the same for field size $14 \times 28 \mathrm{~cm}^{2}$ (largest field size tested) larger than $10 \times 10 \mathrm{~cm}^{2}$ at the CAX of the field. Regarding energies below $200 \mathrm{keV}$ trends were similar with the ones described for depth dependence.

Beyond the effects of field size and application-related variations (e.g. IMRT or split-IMRT spectra) in the spectrum, a number of other features were changing the spectrum accordingly (e.g. spectral shifting of in versus out-of-field area) and were related with the structural elements in the linac head (flattening filter). The most prominent feature was the ubiquitous annihilation peak at $E=511 \mathrm{keV}$, which was more obvious for shallower depths and smaller field sizes due to less scatter and harder spectrum with more high-energy particles.

\section{Spectra variation outside the irradiated field}

Photon energy spectra were also determined for out-of-field areas and compared to the CAX data (Fig. 3). Out-of-field spectra changed significantly with distance from the field edge and that change was larger with depth. At out-of-field positions the beam lacks the uncollided primary component (photons that did not interact and were not scattered), and it had contribution only from head leakage (all photons included those that interacted in the linac head) and radiation scattered inside phantom. As we move away from the CAX the scatter contribution increased and it became the dominant component of the radiation field. Scatter radiation consists of lower energy photons and this was the reason that the aforementioned shifting of the spectrum was observed.

Out-of-field spectra of FFF versus STD linac mainly differentiate below $200 \mathrm{keV}$ and above $500 \mathrm{keV}$. For the lower area FFF spectrum peak value was $\sim 10 \%$ higher than STD linac. In the area between $200 \mathrm{keV}$ and $500 \mathrm{keV}$ spectra were practically the same. As mentioned previously, in the case of FFF beam the overall spectrum was softer than the flattened one. Consequently, the FFF beam had a higher component of low-energy particles as we move away from the treatment field.

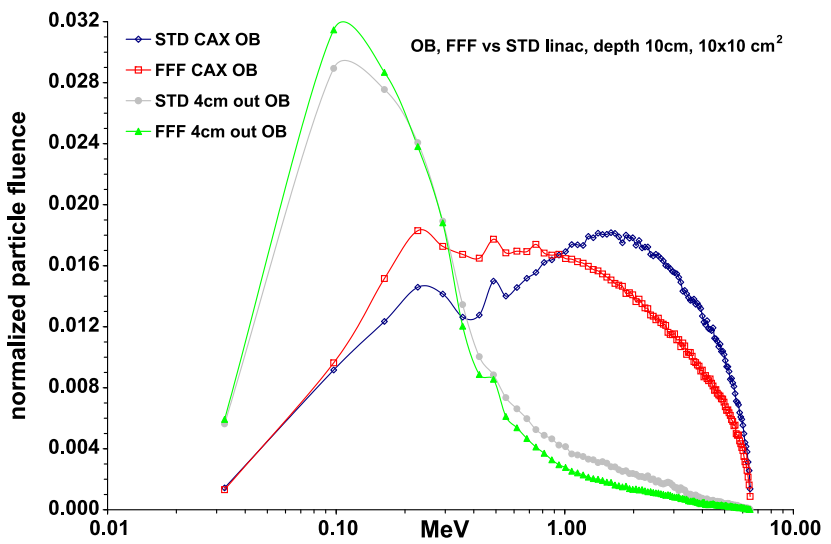

Figure 3. Spectra variation in and out-of-field for open beam (OB) between STD and FFF linac for the case of a $10 \times 10 \mathrm{~cm}^{2}$ at depth of $10 \mathrm{~cm}$. Spectra shown are normalized to the total area. 
Spectral variations for IMRT fields

A comparison was performed for OB fields versus dynamic IMRT fields (dynamic sweeping gaps - SG). Different widths of SG were simulated in order to investigate the potential effect of MLCinduced hardening and scatter on beam spectra. The overall qualitative behavior of IMRT beam spectra was similar to that of OB (Fig. 4). For both conditions, OB and SG, there is a shift of the spectra towards lower energies as we move away from the CAX. The comparison between CAX IMRT spectra and OB showed that there was a hardening in the case of IMRT delivery due to the presence of the MLC leaves in the beam. Hardening can be noticed in the lower $(>200 \mathrm{keV})$ and higher $(>1 \mathrm{MeV})$ spectral regions where the spectrum shifts towards lower energies. The opposite effect can be noticed in the case of out-of-field spectra in the low-energy region.

Although spectral differences between OB and IMRT are small, it was noticeable that the low-energy portion of the spectrum had a larger area under the curve for the OB versus the MLC, owing to the attenuating effect of the latter. Out-of-field IMRT spectral effects between STD and FFF linac were following similar patterns and behavior were practically the same for the lower part of the spectrum with FFF to be $\sim 5 \%$ larger than STD linac. For the high-energy part of the spectrum FFF linac had lower than STD by 15-20\%.

Spectral differences for SG larger than $10 \mathrm{~mm}$ were considered to be negligible as the opening of the SG was large and the scatter from the MLC surfaces did not significantly change the overall spectra compared to the OB. As the SG size was decreased, the spectrum progressively became harder, as the attenuated photons through the MLCs became more dominant, compared to the contribution of laterally scattered photons inside the phantom. The same effect was also presented for the out-of-field spectra. Despite the shift of the spectra towards lower energies there was a significant increase in the fluence for energies larger than $1 \mathrm{MeV}$ due to similar effects.

FFF beams were affected to a lesser extent for larger SG compared to the STD case, as there was already a large contribution from phantom scatter and from the lower energy radiation in the primary beam. Moreover, STD beam is already pre-hardened (due to the presence of the flattening filter), and therefore less prone to exhibit scatter-related spectral variations in different situations. But due to the fact that FFF linac had a lower mean energy and it can be attenuated more as the SG decreases (low-energy photons were absorbed more by the MLCs) or as the distance from the CAX increases (larger phantom scatter due to larger number of lower energy particles of the beam) has a more pronounced hardening from STD one.

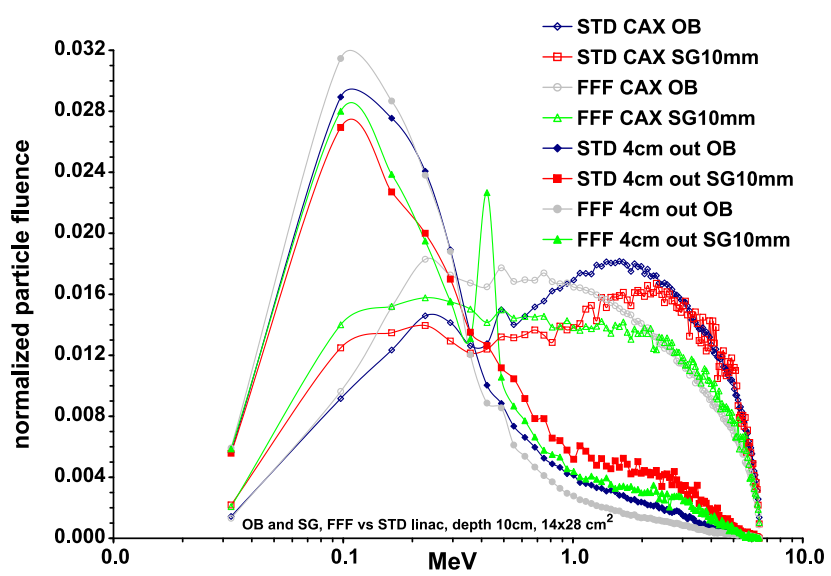

Figure 4. Spectra variation in and out-of-field for open beam (OB) and dynamic IMRT (SG $10 \mathrm{~mm}$ ) fields between STD and FFF linac for the case of a $14 \times 28 \mathrm{~cm}^{2}$ at depth of $10 \mathrm{~cm}$. Spectra shown are normalized to the total area.
Finally, comparison was also performed between irradiation involving two split-IMRT subfields $\left(10 \times 10 \mathrm{~cm}^{2}-\mathrm{SG} 10 \mathrm{~mm}\right)$ and a single IMRT without split (Fig. 1b). The comparison showed that the contribution of the out-of-field dose of the second IMRT subfield on the CAX of the first one softened the STD linac spectrum. In the case of FFF linac the same contribution was not significant as its spectrum was already softer and the low-energy particles contribution from the second IMRT subfield did not affect the spectrum significantly. So, the percentage difference between the split-IMRT and the IMRT spectra, for the energy area below $200 \mathrm{keV}$, was no more than $7 \%$ for the FFF spectra while for STD one was between 50 and 200\% (Fig. 5).

\section{Dose}

In this section, doses arising from the various spectra were analyzed. The impacts of the differential effects seen in the beam quality were also explored.

\section{Absorbed dose variations}

In clinical practice the most important physical parameter is the absorbed dose. Absorbed dose was computed at the same spatial locations as the spectra. Figure 6 shows a bar chart in semilogarithmic scale of the relative absorbed dose for selected simulated cases, computed at the central axis or at $4 \mathrm{~cm}$ outside of the field edges, and normalized to the $\mathrm{cGy} / \mathrm{MU}$ (linac calibrated to deliver $1 \mathrm{Mu}$ per $1 \mathrm{cGy}$ delivered for a $10 \times 10 \mathrm{~cm}^{2}$ open STD beam field at depth of $10 \mathrm{~cm}$ in water). The calculated percentage uncertainty by EGSnrc was not larger than $1.3 \%$ for the case of STD linac for $4 \mathrm{~cm}$ out-of-field. The absorbed dose for FFF linac was larger by a factor of 2 compared with STD linac (FFF-CAX versus STD-CAX and FFF $-4 \mathrm{~cm}$ out versus STD $-4 \mathrm{~cm}$ out) as there was an increased dose rate after removing the flattening filter for the same beam current incident on the linac target. The absorbed dose inside the field, as expected, was approximately one order of magnitude larger than out-of-field for both linac types. However, spectral comparison showed that there is a large increase in the lower energy area of the spectrum as we move away from the CAX due to the increased scatter.

\section{The impact of beam quality on the dose when inhomogeneities are} present

As it was mentioned in Spectrum-weighted dose metrics the spectrum depends on the beam conditions, which impacts the dose

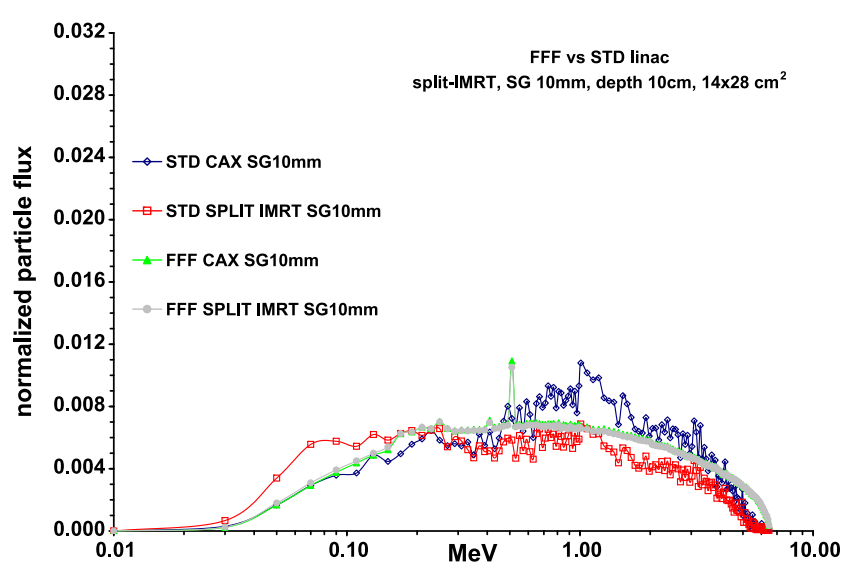

Figure 5. Spectra variation in water for STD and FFF IMRT field $\left(10 \times 10 \mathrm{~cm}^{2}-\mathrm{SG}\right.$ $10 \mathrm{~mm}-d=10 \mathrm{~cm}$ ) versus split-IMRT field for the same field size, depth and sweeping gap size. Spectra shown are normalized to the total area. 


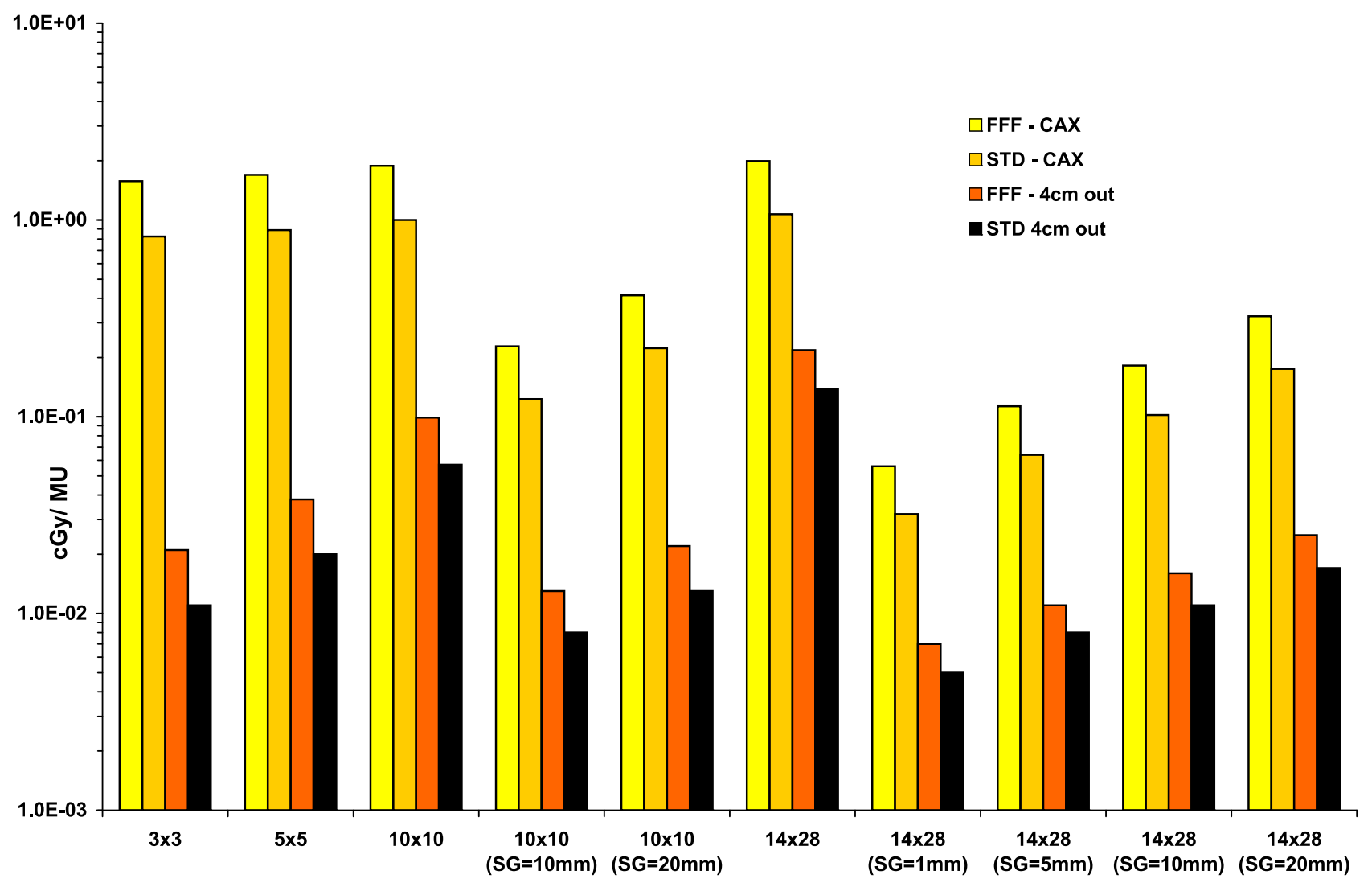

clinical beams

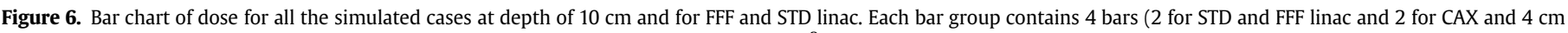

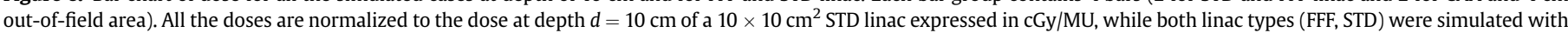
the same initial histories in order, in order to assumed that the beams had the same beam current. Groups without brackets are representing open beam.

deposition. Presence of heterogeneities may have a further significant impact on the dose. Other than normal anatomic features, such heterogeneities may include surgically inserted metals (e.g. a thin plate). In an attempt to evaluate the impact of the spectrum on the dose with or without heterogeneities, two dose metrics, $m(E)$ and $M$, were defined in the Appendix $\mathrm{A}$, and were used here. In considering $m(E)$ and $M$, in addition to their absolute values, the important features were their tendencies and the impact of the differential effects between various irradiation conditions or various depths. The effective dose perturbation factor $M$ increases with the electron density of the material thus it is larger for higher $Z$ and lower energies. In the case of FFF linac photoelectric (PE) absorption dominates because the effective beam energy was lower by about 2 MV compared to the STD linac. Figure 7 shows how the ratio of $\left[\mu_{\mathrm{en}} / \rho\right]_{z} /\left[\mu_{\mathrm{en}} / \rho\right]_{\mathrm{w}}$, which is related to $m(E)$ via Eq (A.7), of a given material $Z$ to water $w$ changes with energy.

The effective dose perturbation metric $M$ increased with depth, field size and $Z$ of the material while the values for FFF linac were larger compared to STD besides a few cases related with the MC noise ( $3 \times 3 \mathrm{~cm}^{2}$ fields size $-4 \mathrm{~cm}$ out-of-field). A similar trend was observed for IMRT versus OB spectra. Figures show $M$ values for all studied cases and for both linac types: FFF (Fig. 8a) and STD (Fig. 8b) and for both mentioned materials gold and $\mathrm{GdO}_{2} \mathrm{~S}_{2}$. Maximum values for both linacs were for gold, out-of-field areas and splitIMRT. Differences between both linacs varied with case and material. FFF linac had in average for all cases $11.3 \%$ higher values for $M$ while for gold was $21.5 \%$ and for $\mathrm{GdO}_{2} \mathrm{~S}_{2}$ was $25 \%$. The largest differences between the two linacs, in respect of the material, were calculated for out-of-field $M$ values where FFF had a higher value of at least $30 \%$.

When the energy-dependent metric $m(E)$ was evaluated it appeared that the metric was mostly sensitive for energies below $\sim 200 \mathrm{keV}$ when it was compared for three different spectral ranges: whole spectrum $\left(E_{\min }, E_{\max }\right)$, and partial integration in the ranges $\left(E_{\min }, 200 \mathrm{keV}\right)$ and $\left(200 \mathrm{keV}, E_{\max }\right)$. The spectrum remains practically the same for energies above $200 \mathrm{keV}$. The calculated difference for the $M$ metric comes from the spectral area below $200 \mathrm{keV}$.

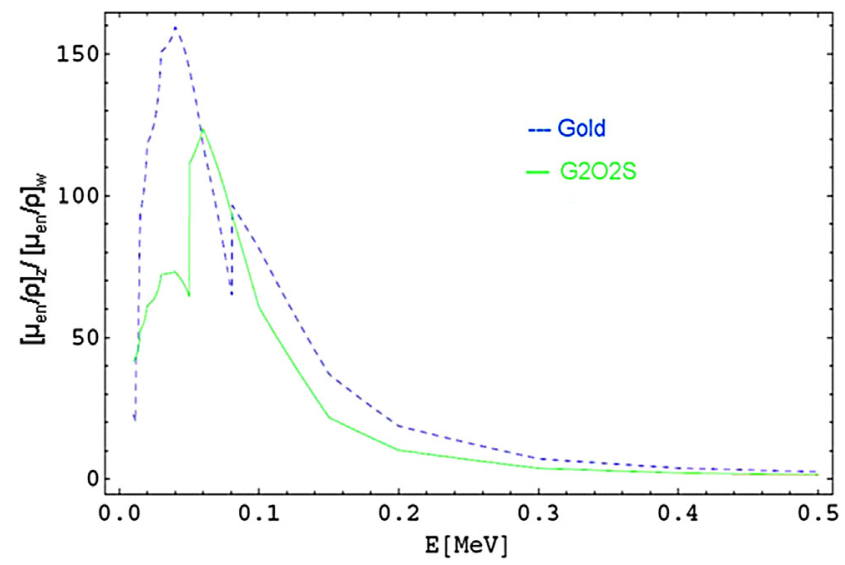

Figure 7. $\left[\mu_{\mathrm{en}} / \rho\right]_{z} /\left[\mu_{\mathrm{en}} / \rho\right]_{\mathrm{w}}$ ratio for two different materials: gold and gadolinium oxysulfide $\left(\mathrm{GdO}_{2} \mathrm{~S}_{2}\right)$ for energy range $10 \mathrm{keV}-500 \mathrm{keV}$. 

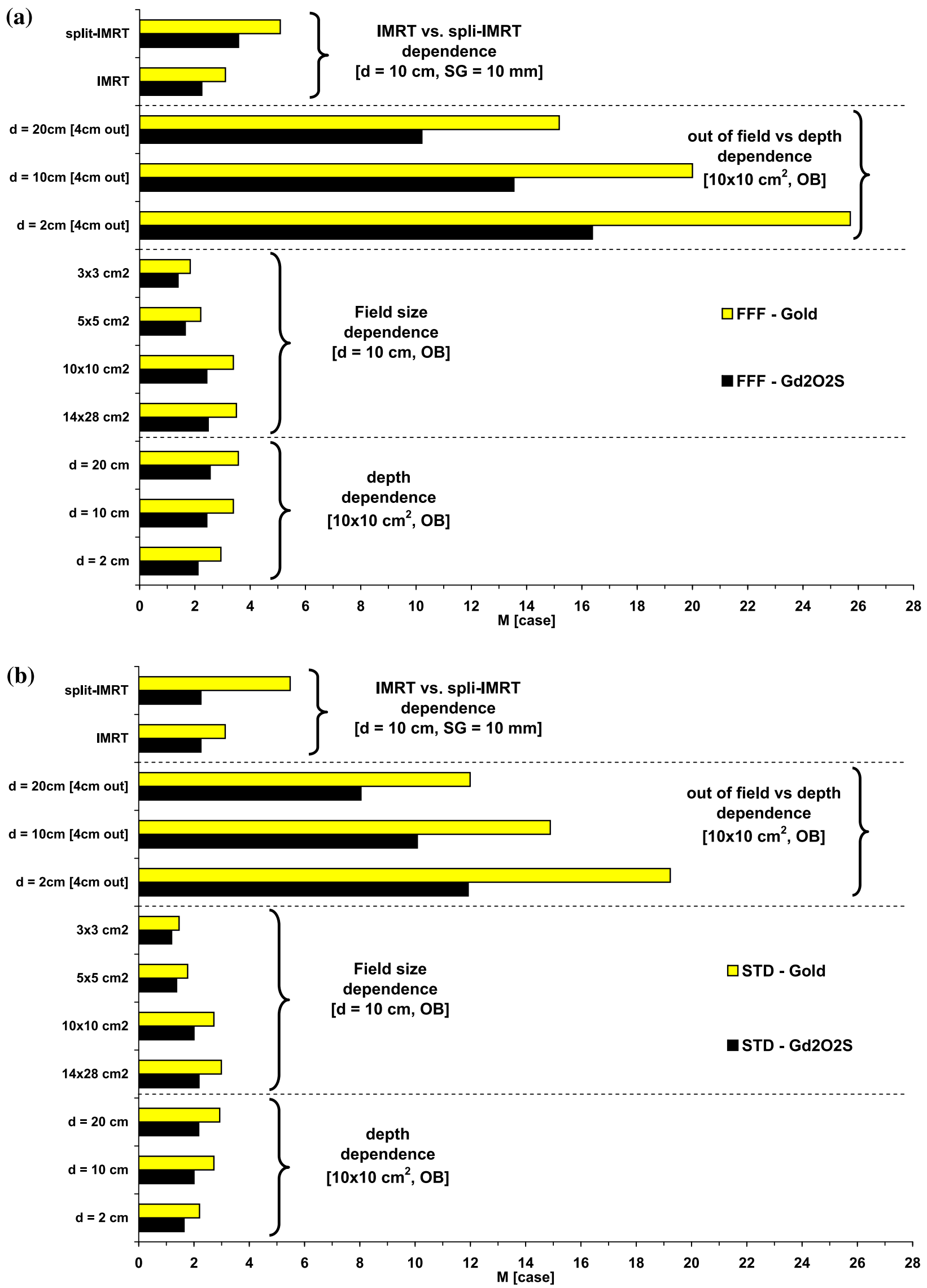

Figure 8. $M$ metric for different clinical spectra for FFF (above - a) and STD (below - b) linac for the case of gold plate and gadolinium oxysulfide $\left(\mathrm{GdO} \mathrm{S}_{2}\right.$ ). 


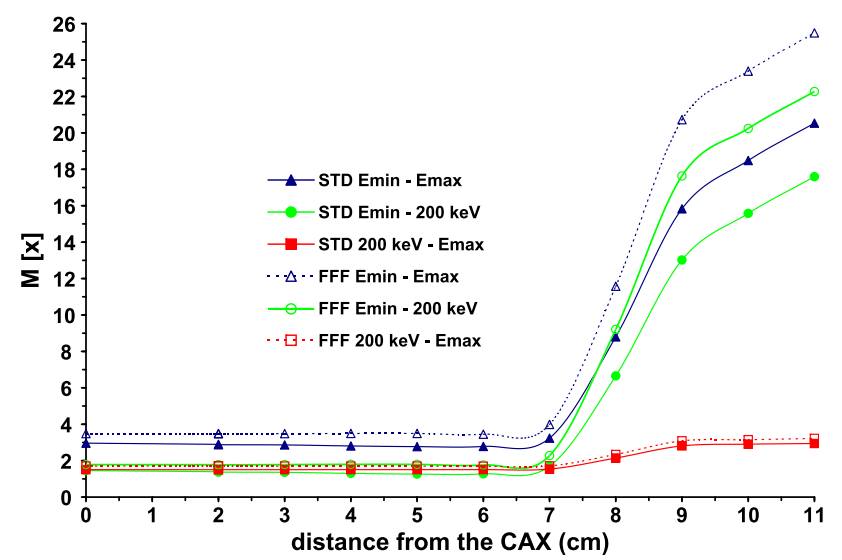

Figure 9. $M[x]$ for gold for the three energy ranges: whole spectrum $\left(E_{\min }-E_{\max }\right), E_{\min }$ $-200 \mathrm{keV}$ and $200 \mathrm{keV}-E_{\max }$ and for STD versus FFF in the case of an OB, $14 \times 28 \mathrm{~cm}^{2}$ field size at depth of $10 \mathrm{~cm}$.

Dose enhanced effect of different spectral areas and their effect on linac with and without the flattening filter can be better demonstrated in Fig. 9. The figure shows how $M\left(x_{i}\right)$ changes from the CAX to $4 \mathrm{~cm}$ out-of-field for points every $1 \mathrm{~cm}$ when gold is present $\left(\mathrm{OB}, 14 \times 28 \mathrm{~cm}^{2}\right)$ for an STD versus an FFF linac.

\section{Discussion}

Uncertainty for spectra calculated at the CAX was below $5 \%$ for all the points except for those at the lowest energies $(<35 \mathrm{keV})$ and the highest energies $(>6.4 \mathrm{MeV})$. Uncertainty in these energy ranges was about $30 \%$ due to the very small number of particles at the edges of the linac spectrum. For out-of-field spectra the uncertainty was below $5 \%$ for $E<3 \mathrm{MeV}$, and $8 \%$ above this energy. Computations at the $511 \mathrm{keV}$ annihilation peak had a much larger uncertainty, $\sim 80 \%$ depending on the case considered. The reason was that the energy binning had to be chosen such as to accommodate this narrow peak without altering the results reported for the rest of the spectrum. Consequently, the magnitude of the annihilation peak should be considered only for qualitative assessments. The high uncertainty experienced in the computation of this particular peak did not affect the rest of the spectrum.

Regardless the different cases studied and the differences shown between FFF and STD linac there were some general trends in the studied spectra related with the underlying physics. Below about $30 \mathrm{keV}$ photons were preferentially absorbed via the PE reaction while higher energy photons undergo Compton scattering. This had lead to a beam hardening at the low-energy end of the spectrum and a simultaneous softening at the mid-range of the spectrum up to about $1 \mathrm{MeV}$. Since the number of incident photons below $30 \mathrm{keV}$ was relatively small, there was a net effective spectrum softening below $1 \mathrm{MeV}$. Above this energy, forward scatter became increasingly dominant with little energy loss; therefore the spectrum between $1 \mathrm{MeV}$ and $6.5 \mathrm{MeV}$ slightly hardened with depth in water. These features can be clearly seen in Figs. 2-4. In addition, there was an increase in the photon fluence between $200 \mathrm{keV}$ and $240 \mathrm{keV}$, which corresponded to the backscatter peak by photons between $1 \mathrm{MeV}$ and $6.5 \mathrm{MeV}$ of incident energy.

In most cases the studied spectrum changed significantly for different clinical beam scenarios. Analysis of all these cases showed that regardless of the specific irradiation conditions (e.g. IMRT versus OB, FFF versus STD linac beam lines, etc. and of course different combinations of those) the spectra can be divided into two major categories: in-field and out-of-field. In most cases FFF spectra had similar shape with STD with the exception of spectral differences with field size and depth. Maxima of the spectra were larger for STD by as much as $40 \%$, with the larger values taking place for smaller field sizes and larger depths and appearing at energies between 1-2 MeV (CAX) and 100-200 keV (out-of-field) depending on the case. In general, the relative changes, between different conditions, were smaller for FFF spectra. However, this was because of the higher component of low-energy photons of the FFF linac (no prehardening effect due to the flattening filter), which already lead to higher dose enhancement than for the STD linac (photon energies were more "uniformly" distributed for FFF spectra and therefore their perturbation resulted in lesser relative changes). As a result of that absolute values of the FFF spectra, without area normalization, were higher than the STD ones.

The largest changes for different beam conditions were observed in the lower energy range, up to $200 \mathrm{keV}$. In all the simulated cases (depth and field dependence, IMRT and split-IMRT, presence or absence of heterogeneities, distance from the CAX) softening of the spectrum was dominant in this area. Differential effects in spectra for various irradiation conditions were not large for energies above $200 \mathrm{keV}$ and can be neglected. FFF spectra were changing following a similar pattern but because their original spectra were softer than those of the STD linac, large differential effects were restricted mostly to the low-energy area $(<200 \mathrm{keV})$. In general, FFF and STD linac spectra can be considered having similar shapes while their integral metric $M$ varied following similar patterns with the relative differences between them.

Since the photon spectrum changes as a function of beam conditions, it was of interest to find a relation between spectral changes and relevant dosimetric quantities. Therefore, we defined two simple dose perturbation metrics: $m(E)$ for each energy bin of the spectra, and $M$, for the whole spectra. Similarly to the analysis of energy fluences for different conditions, attention must be paid to the differential effects in $m(E)$ or $M$, e.g. $\left\{m(E, d)-m\left(E, d_{\text {ref }}\right)\right\}$ or $\left\{M(d)-M\left(d_{\text {ref }}\right)\right\}$ rather than the magnitudes of $M$ or $m(E)$. Here $d$ represents the spatial location (depth in phantom) and $d_{\text {ref }}$ is the reference location.

According to the results presented at Fig. 8a and b dose enhancement applications would be more efficient when FFF linac were used. The benefit might vary on the irradiation conditions, high- $Z$ material used and case studied. Differential effects were mostly due to spectral changes for energies up to about $200 \mathrm{keV}$. For greater energies the spectra remained almost the same. This was particularly true for spectral comparison with depth and field size. The larger the field and depth, the more contribution of the differential effects originate in the low-energy area. In the case of FFF linac, the overall $M$ values were higher, but these differences were coming from the low-energy area, as in the energy range above $200 \mathrm{keV} \mathrm{M}$ had practically the same value as in STD beams.

In the case of out-of-field spectra, $M$ calculation showed that stronger effects. Due to the shifting of the spectrum towards lower energies, integral $M$ values for energies below $200 \mathrm{keV}$ were much higher than in the rest of the spectrum. Differences of $M$ values between the two spectral areas can reach one order of magnitude, depending on the $Z$ of the material (higher for higher $Z$ ). For shallower depths, differences between FFF and STD linac were not as pronounced but as depth increases FFF beams were differentiating less than STD. The reason for that is the same as described above in this section. More low-energy particles are the cause of a "uniformity" in the phantom scatter which was the reason for less relative spectral differences from case to case of FFF versus STD linac.

The case of IMRT for split fields reveals a similar effect. The value of the $M$ metric was practically the same between OB and IMRT for all spectral areas. However, there was a significant increase for the case of the split-IMRT. The contribution of scatter from the split- 
IMRT field leads to a spectrum, which was more susceptible to dose perturbation effects for different materials.

Auger electrons were inherently included in the simulation but because the focus herein is the macroscopic dosimetric quantities the Auger electrons do not have a big impact on them. Auger electrons should increase the dose enhancement ratio (DER) at the microscopic scale (nm). Thus our $m(E)$ is a lower bound for DER if DER is to be calculated at the cell level. As it was mentioned in the Introduction section there were studies showing no clinical significant DER for realistic gold concentrations in case of MV beams [27] as well others showing increase of the DER even by a factor of 8 [24]. The differences in these publications arise from different simulated conditions and normalization schemes used in MC. Clarification of the scale where these effects were calculated as well as normalization of the different quantities used must be done with caution in order comparison between different quantities to be avoided. Our study supports the idea that significant dose enhancement can be achieved in MV beams; especially if the one optimizes the irradiation technique chooses FFF linac.

In the current study IMRT fields were studied by using sweeping gaps. A question exists among medical physicists if these techniques can be used for general conclusion regarding IMRT with real patients. Published studies [31-33] showed that to the first approximation SG can simulate the overall MLC related dose contribution for IMRT field because it was mostly related to the time that the leaves are in the treatment field even though the exact shape of the dose distribution is different locally. The reason for this is that when averaged over larger areas the SG MLC patterns and the patient specific MLC motions result in similar doses and beam qualities. In a clinical plan differences between the inter- and intra-MLC leakage will lead to additional effects such as tongue and groove effect (T\&G) and off-axis dependence of MLC leakage related to different energy of the beam. Due to the complexity of the MLC design and noise in MC simulations, these effects are best characterized and dealt with in the dose calculation algorithm by adopting an empirical approach [31-33], in which special "delayed" SG are used to determine T\&G as well as off-axis dependence of MLC properties for various depths in water. In the presence of high- $Z$ material these effects might be different due to difference in beam quality.

\section{Conclusions}

Spectral differences between STD and FFF 6 MV beam lines were studied for an extensive number of irradiation conditions. Reference data for these clinical beams were produced followed by qualitative and quantitative analysis of differential effects observed in different spectral regions or in the effective beam quality for different high- $Z$ materials.

In the past few years there have been an increased number of publications regarding dose enhancement effects. As our study implies, that the knowledge spectral beam properties of various irradiation techniques (FFF versus STD or OB versus IMRT) in different irradiation areas (in- versus out-of-field), is essential because relatively large discrepancies in dose enhancement exist. Moreover, accumulation of high- $Z$ contrast agent in tumor as well as in normal tissue has to be taken into account because of the lowenergy of scattered photons.

Qualitative analysis revealed similar patterns and shapes between spectra for STD and FFF linacs, with FFF beams being softer due to the lack of flattening filter to attenuate the low-energy component of the beam. There was a progressive shift of the spectra towards lower energies for both linac types with increased depth, field size and distance. The greatest dose enhancement was obtained for FFF linac beams, split-IMRT fields and deep seated tumors.

In the second part of the spectral analysis the relationship between spectral changes and dose was investigated. Different spectral scenarios lead to different dose perturbations, which increase for higher $Z$ materials. The differential effect was directly related to the low-energy part of the spectrum (below $200 \mathrm{keV}$ ). For the remaining part of the spectrum $(>200 \mathrm{keV})$, the metric $M$ remained almost constant and equal to 1 .

\section{Acknowledgments}

The authors would like to thank Varian Medical Systems for providing the blueprints for the Monte Carlo simulations. This research was partially supported by Kayes/JCRT awards.

\section{Appendix A}

Consider heterogeneity embedded in water phantom, which was exposed to a photon fluence of $\Phi(E)$. Two simple metrics of dose perturbation (enhancement or suppression) may be defined due to the presence of the embedded medium: a differential metric $m(E)$, which is energy-dependent, and an integral or effective metric, $M$, which are related as:

$M=\int_{0}^{\infty} m(E) \mathrm{d} E$.

In practice, the upper limit of the above integral may be replaced by the maximum energy of the incident particles, $E_{\max }$, assuming there is no up-scattering. For megavoltage linacs this condition is satisfied. For a material of different composition than water (which may be an aqueous mixture or solution, denoted here by the letter $Z$ ), inserted in a water phantom (denoted by $w$ ), the differential metric is the ratio of the energy-dependent dose in material $Z$ versus the total dose in water at the same spatial location, $r_{0}$, in the absence of material $Z$ :

$m\left(r_{0}, E\right)=\frac{D_{Z}\left(r_{0}, E\right)}{D_{\mathrm{w}}\left(r_{0}\right)}$,

and

$M\left(r_{0}\right)=\frac{D_{Z}\left(r_{0}\right)}{D_{\mathrm{w}}\left(r_{0}\right)}$

where $D_{Z}$ and $D_{\mathrm{w}}$ are the total doses summed overall energies in the spectrum in material $Z$ and in water without the presence of $Z$, respectively:

$D_{Z}=\int_{0}^{\infty} D_{Z}(E) \mathrm{d} E$ and $D_{\mathrm{w}}=\int_{0}^{\infty} D_{\mathrm{w}}(E) \mathrm{d} E$

Since the spatial location is fixed, it can be dropped from the notation to preserve clarity. In charged particle equilibrium (CPE) the dose may be computed using the collisional kerma:

$D(E) \stackrel{\mathrm{CPE}}{=} E \Phi(E) \frac{\mu_{\mathrm{en}}(E)}{\rho}=\Psi(E) \frac{\mu_{\mathrm{en}}(E)}{\rho}$

where $\Psi(E)=E \Phi(E)$ is the energy fluence and $\mu_{\mathrm{en}} / \rho$ is the massenergy absorption coefficient. Hence in CPE the integral and differential dose perturbation metrics may be written as 


$$
M=\frac{D_{Z}}{D_{\mathrm{w}}} \stackrel{\text { CPE }}{=} \frac{\int_{0}^{E_{\max }} \Psi_{Z}(E)\left[\mu_{\mathrm{en}}(E) / \rho\right]_{Z} \mathrm{~d} E}{\int_{0}^{E_{\max }} \Psi_{\mathrm{w}}(E)\left[\mu_{\mathrm{en}}(E) / \rho\right]_{\mathrm{w}} \mathrm{d} E}
$$

and

$$
m(E)=\frac{D_{Z}(E)}{D_{\mathrm{w}}} \stackrel{\mathrm{CPE}}{=} \frac{\Psi_{Z}(E)\left[\mu_{\mathrm{en}}(E) / \rho\right]_{Z}}{\int_{0}^{E_{\max }} \Psi_{\mathrm{w}}(E)\left[\mu_{\mathrm{en}}(E) / \rho\right]_{\mathrm{w}} \mathrm{d} E},
$$

respectively. In the above formula, the upper limits of the energy integrals are replaced by the maximum energy of the incident particles, $E_{\max }$.

A simplification may be afforded by the assumption that if the electron density in material $Z$ is not substantially different from that in water, the photon spectra in these materials will also not differ substantially, hence $\Phi_{\mathrm{Z}}(E) \sim \Phi_{\mathrm{w}}(E)$ and the subscripts for $\Psi_{Z}(E)$ and $\Psi_{\mathrm{w}}(E)$ in Eqs. (A.6) and (A.7) may be dropped. A similar argument can be made for the case when material $Z$ is a thin solid embedded in the water phantom, as the fluence is continuous across the interface of $Z$ and w. Under this assumption Eqs. (A.6) and (A.7) holds. Strictly speaking the quantities, which we refer to as metrics $M$ and $m(E)$ are not exactly dose ratios. They are approximately primary dose ratios under conditions of CPE and when the primary collision kerma can be used to represent the energy deposition. Therefore, we refer to them as metrics of dose effects rather than true dose ratios. In case of a mixture of high- $Z$ contrast agent with tissue the above equations can be extended to a new concentration-weighted $m(E)$ value $\left[\left(\mu_{\mathrm{en}} / \rho\right)\right.$ following NIST [30] calculation of mixtures].

\section{References}

[1] Liu HH, Verhaegen F. An investigation of energy spectrum and linear energy variations in mega-voltage photon beams used in radiotherapy. Radiat Prot Dosim 2002;99(1-4):425-7.

[2] Scarboro SB, Followill DS, Howell RM, Kry SF. Variations in photon energy spectra of a 6 MV beam and their impact on TLD response. Med Phys 2011;38(5):2619-28.

[3] Tsechanski A, Krutman Y, Faermann S. On the existence of low-energy photons $(<150 \mathrm{keV})$ in the unflattened $\mathrm{X}$-ray beam from an ordinary radio therapeutic target in a medical linear accelerator. Phys Med Biol 2005;50: 5629-39.

[4] Jang SY, Liu HH, Mohan R, Siebers JV. Variations in energy spectra and waterto-material stopping-power ratios in three-dimensional conformal and intensity-modulated photon fields. Med Phys 2007;34:1388-97.

[5] Ding GX. Energy spectra, angular spread, fluence profiles and dose distributions of 6 and $18 \mathrm{MV}$ photon beams: results of Monte Carlo simulations for a Varian 2100EX accelerator. Phys Med Biol 2002;47:1025-46.

[6] Faddegon BA, Wu V, Pouliot J, Gangadharan B, Bani-Hashemi A. Low dose megavoltage cone beam computed tomography with an unflattened $4 \mathrm{MV}$ beam from a carbon target. Med Phys 2008;35(12):5777-86.

[7] Sawkey M, Lu O, Morin M, Aubin S, Yom S, Gottschalk AR, et al. A diamond target for megavoltage cone-beam CT. Med Phys 2010;37(3):1246-53.

[8] Han Z, Ng SK, Bhagwat MS, Lyatskaya Y, Zygmanski P. Evaluation of MatriXX for IMRT and VMAT dose verifications in peripheral dose regions. Med Phys 2010;37:3704-14.
[9] Titt U, Vassiliev ON, Pönisch F, Dong L, Liu H, Mohan R. A flattening filter free photon treatment concept evaluation with Monte Carlo. Med Phys 2006;33: 1595-602.

[10] Vassiliev ON, Titt U, F Kry S, Pönisch F, Gillin MT, Mohan R. Monte Carlo study of photon fields from a flattening filter-free clinical accelerator. Med Phys 2006;33:820-7.

[11] Vassiliev ON, Titt U, Kry SF, Mohan R, Gillin M. Dosimetric properties of photon beams from a flattening free clinical accelerator. Phys Med Bio 2006;51:1907-17.

[12] önisch FP, Titt U, Vassiliev ON, Kry SF, Mohan R. Properties of unflattened photon beams shaped by a multileaf collimator. Med Phys 2006;33:1738-46.

[13] Zhu XR, Kang Y, Gillin MT. Measurements of in-air output ratios for a linear accelerator with and without the flattening filter. Med Phys 2006;33: 3723-33.

[14] Titt U, Vassiliev ON, Pönisch F, Kry SF, Mohan R. A Monte Carlo study of backscatter in a flattening filter free clinical accelerator. Med Phys 2006;33: 3270-3.

[15] Cashmore J. The characterization of unflattened photon beams from a $6 \mathrm{MV}$ linear accelerator. Phys Med Biol 2008;53:1933-46.

[16] Xiong G, Rogers DWO. Relationship between \%dd(10) $x$ and stopping-power ratios for flattening filter free accelerators: a Monte Carlo study. Med Phys 2008;35:2104-9.

[17] Kragl G, Wetterstedt $S$ af, Knäusl B, Lind $M$, McCavana $P$, Knöös $T$, et al. Dosimetric characteristics of 6 and 10 MV unflattened photon beams. Radiother Oncol 2009;93:141-6.

[18] Ceberg C, Johnsson S, Lind M, Knöös T. Prediction of stopping-power ratios in flattening-filter free beams. Med Phys 2010;37:1164-8.

[19] Kry SF, Vassiliev ON, Mohan R. Out-of-field photon dose following removal of the flattening filter from a medical accelerator. Phys Med Biol 2010;55: 2155-66.

[20] Georg D, Kragl G, Wettersterdt S af, McCavana P, McClean B, Knoos T. Photon beam quality variations of a flattening filter free linear accelerator. Med Phys 2010;37:49-53.

[21] Tsiamas P, Seco J, Han Z, Bhagwat M, Maddox J, Kappas C, et al. A modification of flattening filter free linac for IMRT. Med Phys 2011;38(5):2342-52.

[22] Cho SH. Estimation of tumor dose enhancement due to gold nanoparticles during typical radiation treatments: a preliminary Monte Carlo study. Phys Med Biol 2005;50:N163.

[23] Cho SH, Jones BL, Krishnan S. The dosimetric feasibility of gold nanoparticleaided radiation therapy (GNRT) via brachytherapy using low-energy gamma-/ X-ray sources. Phys Med Biol 2009;54:4889-905.

[24] Jones B, Krishnan S, Cho SH. Estimation of microscopic dose enhancement factor around gold nanoparticles by Monte Carlo calculations. Med Phys 2010;37(7):3809-16.

[25] Leung MKK, Chow JCL, Chithrani BD, Lee MJG, Oms B, Jaffray DA. Irradiation of gold nanoparticles by X-rays: Monte Carlo simulation of dose enhancements and the spatial properties of the secondary electrons production. Med Phys 2011;38(2):624-31.

[26] Van den Heuvel F, Locquet J-P, Nuyts S. Beam energy considerations for gold nano-particle enhanced radiation treatment. Phys Med Biol 2010;55 4509-20.

[27] Lechtman E, Chattopadhyay N, Cai Z, Mashouf S, Reilly R, Pignol JP. Implications on clinical scenario of gold nanoparticle radiosensitization in regards to photon energy, nanoparticle size, concentration and location. Phys Med Biol 2011;56:4631-47.

[28] Kawrakow I, Mainegra-Hing E, Rogers DWO. EGSnrcMP: the multi-platform environment for EGSnrc. NRCC Report PIRS-877; 2006.

[29] Karnas SJ, Yu E, McGarry RC, Battista JJ. Optimal photon energies for IUdR Kedge radiosensitization with filtered $\mathrm{X}$-ray and radioisotope sources. Phys Med Biol 1999;44(10):2537-49.

[30] Hubbell JH, Seltzer SM. Tables of X-ray mass attenuation coefficients and mass energy-absorption coefficients from $1 \mathrm{keV}$ to $20 \mathrm{MeV}$ for elements $\mathrm{z}=1$ to 92 and 48 additional substances of dosimetric interest. NIST. http://www.nist. gov/pml/data/xraycoef/index.cfm; 2009.

[31] Zygmanski P, Rosca F, Kadam D, Lorenz F, Nalichowski A, Court L, et al. Determination of depth and field size dependence of MLC transmission in IMRT beams. J Appl Clin Med Phys 2007;8:76-95.

[32] Lorenz F, Nalichowski A, Rosca F, Killoran J, Wenz F, Zygmanski P. An independent dose calculation algorithm for MLC-based radiotherapy including the spatial dependence of mlc transmission. Phys Med Biol 2008;53:557-73.

[33] Lorenz F, Nalichowski A, Rosca F, Kung J, Wenz F, Zygmanski P. Spatial dependence of mlc transmission in IMRT delivery. Phys Med Biol 2007;52: 5985-99. 